\title{
Tibiopedal arterial minimally invasive retrograde revascularization (TAMI) in patients with peripheral arterial disease and critical limb ischemia. On behalf of the Peripheral Registry of Endovascular Clinical Outcomes (PRIME)
}

\author{
Jihad A. Mustapha MD ${ }^{1}$ | Fadi Saab MD ${ }^{1}$ | Theresa N. McGoff BSN ${ }^{2}$ | \\ George Adams MD ${ }^{3}$ | John R. Mullins MD ${ }^{4}$ | Ashraf Al-Dadah MD ${ }^{5}$ | \\ Michael R. Jaff DO ${ }^{6}$ | Philip P. Goodney MD M $^{7}$ Farhan Khawaja MD ${ }^{8}$ | \\ Larry J. Diaz-Sandoval MD ${ }^{2}$
}

${ }^{1}$ Advanced Cardiac \& Vascular Centers for Amputation Prevention, Grand Rapids, Michigan

${ }^{2}$ Metro Health-University of Michigan Health, Wyoming, Michigan

${ }^{3}$ Rex Healthcare and University of North Carolina Health Systems, Raleigh,

North Carolina

${ }^{4}$ Cox Health, Springfield, Missour

${ }^{5}$ Prairie Heart Institute, Springfield, Illinois

${ }^{6}$ Newton Wellesley Hospital, Boston,

Massachusetts

${ }^{7}$ Dartmouth-Hitchcock Medical Center, New Hampshire, Lebanon

${ }^{8}$ Orlando Health, Orlando, Florida

\section{Correspondence}

Larry J. Diaz-Sandoval, Metro HealthUniversity of Michigan Health, 5900 Byron Center Ave, SW, Wyoming, MI 49519.

Email: larry.diaz@metrogr.org

Funding information

BARD; Boston Scientific Corporation

Cardiovascular Systems, Inc; Terumo

\begin{abstract}
Objectives and background: Complex peripheral arterial disease (PAD) and critical limb ischemia (CLI) are associated with high morbidity and mortality. Endovascular techniques have become prevalent in treatment of advanced PAD and CLI, and use of techniques such as tibiopedal minimally invasive revascularization (TAMI), have been proven safe in small, single-center series. However, its use has not been systematically compared to traditional approaches.
\end{abstract}

Methods and results: This is a retrospective, multicenter analysis which enrolled 744 patients with advanced PAD and CLI who underwent 1,195 endovascular interventions between January 2013 and April 2018. Data was analyzed based on access used for revascularization: 840 performed via femoral access, 254 via dual access, and 101 via TAMI. The dual access group had the highest median Rutherford Class and lowest number of patent tibial vessels. Median fluoroscopy time, procedure time, hospital stay, and contrast volume were significantly lower in the TAMI access group when compared to both femoral/dual access groups. There was also a significant difference between all groups regarding location of target lesions: Femoropopliteal lesions were most commonly treated via femoral access; infrapopliteal lesions, via TAMI, and multilevel lesions via dual access.

Conclusions: Stand-alone TAMI or tibial access as an integral part of a dual access treatment strategy, is safe and efficacious in the treatment of patients with advanced PAD and CLI who have infrapopliteal lesions. Larger prospective and randomized studies may be useful to further validate this approach.

\section{KEYWORDS}

critical limb ischemia, endovascular, peripheral artery disease, radiation safety, tibial access 


\section{1 | INTRODUCTION}

Peripheral artery disease (PAD) is a worldwide epidemic, which impacts one in 10 patients over the age of 70 years. ${ }^{1}$ Critical limb ischemia (CLI) represents the most advanced stage of $P A D$, and is defined as the presence of rest pain and/or ulceration of the feet. Patients with CLI have a significant risk of amputation due to disruption of the distal microcirculation and its ability to provide nutrient-rich arterial blood flow to the lower extremity end-organ. Patients with PAD and CLI tend to suffer from significant comorbidities including cerebrovascular and coronary artery disease, diabetes, chronic renal failure, hypertension, dyslipidemia, and tobacco abuse. ${ }^{2-4}$ These comorbidities not only worsen the prognosis of these patients but may also limit the patient's ability to undergo surgical revascularization.

As such, endovascular revascularization techniques have continued to increasingly become the most common therapeutic choice when compared to open surgical approaches. ${ }^{5}$ This trend is in part a reflection of the predominant frailty characteristic of CLI patients (which makes them less suitable candidates for surgery), and a consequence of the increased availability of endovascular technologies coupled with a rapid adoption and evolving mastery of these new devices and techniques. However, even endovascular therapy (EVT) may prove challenging to perform on a number of patients. Limitations include inadequate arterial access sites due to severely diseased common femoral artery conduits, inability of the patients to lay flat, severe morbid obesity, infected groins, and/or increased risk for contrast-induced nephropathy in patients with baseline chronic kidney disease. The Tibiopedal Arterial Minimally Invasive (TAMI) retrograde revascularization technique is an innovative modality for tibiopedal access and treatment, ${ }^{6}$ that seeks to circumvent some of these limitations.

Since its introduction in 2013, the TAMI technique has been used to provide treatment for patients lacking the ability to be treated from an antegrade femoral and/or contralateral retrograde femoral approach (due to the aforementioned reasons). Thus far, no analysis has been done comparing the use of the TAMI technique with the more traditional endovascular access approaches, nor are we aware of any outcomes data. This study seeks to examine and compare the use of this strategy and its outcomes versus those obtained in patients who underwent revascularization via the traditional femoral access approach or the dual access approach (antegrade ipsilateral femoral or retrograde contralateral femoral, combined with retrograde tibiopedal access).

\section{2 | METHODS}

\section{$2.1 \mid$ Subjects}

This is a retrospective analysis of prospectively collected data, as part of a multicenter study of consecutive PAD and $C L I$ subjects who underwent lower extremity endovascular revascularization in five centers in the United States between January 2013 and April 2018, as part of the Peripheral Reglstry of Endovascular Clinical OutcoMEs
(PRIME Registry). ${ }^{7}$ Institutional Review Board approval was obtained at each institution and subject consents were attained prior to any procedures or data collection. Eligible subjects were adults $\geq 18$ years with symptomatic PAD (Rutherford class 3 ) and CLI (Rutherford class 4-6) undergoing EVT of a lower extremity.

\section{2 | Procedure}

Endovascular revascularization was attempted on all study subjects. Access type was determined by the treating physician and included one of the following: ipsilateral common femoral or superficial femoral antegrade access; contralateral common femoral retrograde access; dual access (common femoral antegrade/retrograde access + tibial pedal access), or TAMI access (retrograde tibial-pedal access alone with the intention to carry the intervention from this access site). Revascularization methods were also determined by the treating physician and included one or a combination of the following: atherectomy, percutaneous transluminal angioplasty (PTA), drug-coated balloon angioplasty (DCB), bare-metal stent (BMS) or drug-eluting stent (DES) placement.

\subsection{Outcomes and definitions}

Postrevascularization angiography was performed to assess treatment success, which was defined as $\leq 30 \%$ final residual stenosis.

Complications were defined as the occurrence of any of the following in the treated vessel and/or at the access site: arterio-venous fistula, thrombus, aneurysm, pseudoaneurysm, hematoma (defined as subcutaneous blood collection with induration measuring more than $3 \mathrm{~cm}$ in diameter), infection, retroperitoneal bleed, compartment syndrome, postprocedural need for blood transfusion, unplanned above the ankle amputation, embolization, dissection, and persistent vasospasm. Perforations were classified as mild, moderate and severe. Mild perforations were defined as delayed extravasation after contrast injection, with uninterrupted blood flow beyond the extravasation site, which resolves with prolonged balloon inflation. Moderate was defined as immediate contrast extravasation, with uninterrupted blood flow beyond the extravasation site, which resolves with prolonged balloon inflation. Severe was defined as immediate contrast extravasation, with interrupted blood flow beyond the extravasation site, which requires treatment with covered stents or coils. Vessel rupture was defined as immediate contrast extravasation which requires open surgical intervention for repair.

Complication-free result was defined as no complications between the end of the procedure and the time of patient discharge.

\subsection{Data analysis}

Categorical variables were reported as counts and percentages and continuous variables were reported as median and interquartile range 
(IQR). Three groups were analyzed: Femoral access, Dual access, and TAMI access. Two-sided omnibus $p$-values for group differences, were derived from Fisher's exact test for categorical variables and KruskalWallis analysis of variance for continuous variables. When group differences were identified by an omnibus $p$-value less than .05 , Bonferroni post hoc tests were performed among all pairwise comparisons. Statistical significance was defined as $p<.01$. Data were analyzed with SAS v9.4 (SAS Institute, Cary, NC).

\section{3 | RESULTS}

A total of 744 subjects clinically diagnosed with PAD and CLI underwent 1,195 endovascular lower extremity revascularization procedures. Subject data was analyzed based on the type of access method used for revascularization: 840 interventions were carried via a femoral (ipsilateral antegrade or contralateral retrograde) access, 254 interventions were carried via dual access (femoral + tibial-pedal), and

TAB LE 1 Comparison of patient characteristics by access type at index procedure

\begin{tabular}{|c|c|c|c|c|c|}
\hline \multirow[b]{2}{*}{ Characteristic } & \multicolumn{3}{|l|}{ Access type } & \multicolumn{2}{|c|}{ Significance $^{a}$} \\
\hline & $\begin{array}{l}\text { TAMI } \\
\text { (46 patients) }\end{array}$ & $\begin{array}{l}\text { Femoral } \\
\text { (546 patients) }\end{array}$ & $\begin{array}{l}\text { Dual access } \\
\text { (152 patients) }\end{array}$ & $\begin{array}{l}\text { Omnibus } \\
p \text {-value }\end{array}$ & $\begin{array}{l}\text { Bonferroni } \\
\text { Post hoc }\end{array}$ \\
\hline \multicolumn{6}{|l|}{ Demographics } \\
\hline Age, y & 65.3 (59.4-78.3) & $69.8(61.9-77.4)$ & $72.5(64.2-79.1)$ & .03 & b \\
\hline Male gender & $67.4 \%(31 / 46)$ & $61.4 \%(335 / 546)$ & $73.0 \%(111 / 152)$ & .03 & c \\
\hline Race & & & & .76 & \\
\hline White & $91.3 \%(42 / 46)$ & $91.4 \%(499 / 546)$ & $93.4 \%(142 / 152)$ & & \\
\hline Black & $8.7 \%(4 / 46)$ & $6.6 \%(36 / 546)$ & $5.9 \%(9 / 152)$ & & \\
\hline Other & $0.0 \%(0 / 460)$ & $2.0 \%(11 / 546)$ & $0.7 \%(1 / 152)$ & & \\
\hline Body mass index, $\mathrm{kg} / \mathrm{m}^{2}$ & $31.4(25.6-35.1)$ & $28.4(25.0-32.7)$ & $28.6(25.8-32.7)$ & .10 & \\
\hline Insurance & & & & .28 & \\
\hline Medicare & $71.7 \%(33 / 46)$ & $73.3 \%(400 / 546)$ & $79.6 \%(121 / 152)$ & & \\
\hline Private & $15.2 \%(7 / 46)$ & $20.0 \%(109 / 546)$ & $14.5 \%(22 / 152)$ & & \\
\hline Medicaid & $13.0 \%(6 / 46)$ & $5.9 \%(32 / 546)$ & $5.9 \%(9 / 152)$ & & \\
\hline Self-pay/no insurance & $0.0 \%(0 / 46)$ & $0.9 \%(5 / 546)$ & $0.0 \%(0 / 152)$ & & \\
\hline \multicolumn{6}{|l|}{ Medical history } \\
\hline Hypertension & $95.7 \%(44 / 46)$ & $85.7 \%(468 / 546)$ & $88.2 \%(134 / 152)$ & .14 & \\
\hline Peripheral artery disease & $93.5 \%(43 / 46)$ & $92.7 \%(506 / 546)$ & $92.8 \%(141 / 152)$ & $>.99$ & \\
\hline Dyslipidemia & $91.3 \%(42 / 46)$ & $83.2 \%(454 / 546)$ & $90.8 \%(138 / 152)$ & .03 & b \\
\hline Diabetes mellitus & $67.4 \%(31 / 46)$ & $53.3 \%(291 / 546)$ & $57.9 \%(88 / 152)$ & .14 & \\
\hline Smoking history & $65.2 \%(30 / 46)$ & $81.0 \%(442 / 546)$ & $73.7 \%(112 / 152)$ & .01 & b \\
\hline Coronary artery disease & $54.3 \%(25 / 46)$ & $54.2 \%(296 / 546)$ & $59.2 \%(90 / 152)$ & .55 & \\
\hline Prior endovascular procedure & $37.0 \%(17 / 46)$ & $40.3 \%(220 / 546)$ & $44.7 \%(68 / 152)$ & .53 & \\
\hline Prior coronary intervention & $28.3 \%(13 / 46)$ & $30.0 \%(164 / 546)$ & $30.9 \%(47 / 152)$ & .95 & \\
\hline Myocardial infarction & $26.1 \%(12 / 46)$ & $22.3 \%(122 / 546)$ & $19.1 \%(29 / 152)$ & .53 & \\
\hline Coronary artery bypass graft & $21.7 \%(10 / 46)$ & $21.1 \%(115 / 546)$ & $31.1 \%(47 / 151)$ & .04 & c \\
\hline COPD & $17.4 \%(8 / 46)$ & $19.2 \%(105 / 546)$ & $11.2 \%(17 / 152)$ & .06 & \\
\hline Congestive heart failure & $15.2 \%(7 / 46)$ & $16.7 \%(91 / 545)$ & $25.0 \%(38 / 152)$ & .06 & \\
\hline Atrial fibrillation & $15.2 \%(7 / 46)$ & $11.9 \%(65 / 546)$ & $20.7 \%(31 / 150)$ & .02 & c \\
\hline Cerebrovascular disease & $13.0 \%(6 / 46)$ & $14.0 \%(76 / 544)$ & $19.1 \%(29 / 152)$ & .28 & \\
\hline Angina & $6.5 \%(3 / 46)$ & $11.2 \%(61 / 546)$ & $9.3 \%(14 / 150)$ & .64 & \\
\hline Dialysis & $2.2 \%(1 / 46)$ & $4.6 \%(25 / 546)$ & $7.3 \%(11 / 151)$ & .34 & \\
\hline
\end{tabular}

Note: Categorical variables are listed as percent $(n / N)$; continuous variables are listed as median (interquartile range).

Abbreviations: ANOVA, analysis of variance; COPD, chronic obstructive pulmonary disease; TAMI, tibiopedal arterial minimally invasive.

${ }^{a}$ Omnibus $p$-value derived from Fisher's exact test for categorical variables and Kruskal-Wallis ANOVA for continuous variables. Bonferroni post hoc tests were performed among all pairwise comparisons for variables with omnibus $p$-value <.05.

${ }^{\mathrm{b}}$ No statistically significant difference among any pairwise comparison.

'Statistically significant difference between Femoral and Dual Access groups. 
TAB LE 2 Procedure and recovery data by access type

\begin{tabular}{|c|c|c|c|c|c|}
\hline Characteristic & \multicolumn{3}{|l|}{ Access type } & \multicolumn{2}{|c|}{ Significance $^{a}$} \\
\hline Peak ACT, s & 205 (183-239) & $210(188-242)$ & $223(202-246)$ & $<.001$ & $b, d$ \\
\hline Contrast volume, $\mathrm{ml}$ & $50(30-75)$ & $160(118-216)$ & $160(115-220)$ & $<.001$ & $c, d$ \\
\hline Procedure time, min & $64(47-86)$ & 74 (53-99) & $124(95-156)$ & $<.001$ & $b, c, d$ \\
\hline Hospital stay, d & $0(0-1)$ & $1(1-1)$ & $1(1-1)$ & $<.001$ & $b, c, d$ \\
\hline Hospital stay $\geq 2 \mathrm{~d}$ & $7.9 \%(8 / 101)$ & $13.1 \%(110 / 840)$ & $16.9 \%(43 / 254)$ & .07 & \\
\hline
\end{tabular}

Note: Variables are listed as median (interquartile range).

Abbreviations: ANOVA, analysis of variance; TAMI, tibiopedal arterial minimally invasive.

${ }^{a}$ Omnibus $p$-value derived from Kruskal-Wallis ANOVA. Bonferroni post hoc tests were performed among all pairwise comparisons for variables with omnibus $p$-value $<.05$.

${ }^{\text {b }}$ Statistically significant difference between Femoral and Dual Access groups.

${ }^{\mathrm{c}}$ Statistically significant difference between TAMI and Femoral groups.

${ }^{\mathrm{d} S}$ Statistically significant difference between TAMI and Dual Access groups.

TAB LE 3 Vascular access data by access type

\begin{tabular}{|c|c|c|c|c|c|}
\hline Characteristic & \multicolumn{3}{|l|}{ Access type } & \multicolumn{2}{|c|}{ Significance $^{a}$} \\
\hline $4 \mathrm{Fr}$ & $100 \%(106 / 106)$ & $8.6 \%(81 / 941)$ & $52.3 \%(274 / 524)$ & & \\
\hline $5 \mathrm{Fr}$ & $0.0 \%(0 / 106)$ & $81.3 \%(765 / 941)$ & $42.0 \%(220 / 524)$ & & \\
\hline $7 \mathrm{Fr}$ & $0.0 \%(0 / 106)$ & $3.6 \%(34 / 941)$ & $0.4 \%(2 / 524)$ & & \\
\hline Ultrasound guidance & $99.1 \%(106 / 107)$ & $87.7 \%(835 / 952)$ & $97.2 \%(523 / 538)$ & $<.001$ & $\mathrm{~b}, \mathrm{c}$ \\
\hline Access success & $100 \%(107 / 107)$ & $99.1 \%(943 / 952)$ & $100 \%(538 / 538)$ & .06 & \\
\hline Access success on first attempt & $77.6 \%(83 / 107)$ & $84.4 \%(786 / 931)$ & $76.8 \%(411 / 535)$ & $<.001$ & b \\
\hline Time to access, $\mathrm{s}$ & $42(15-98)$ & $40(15-99)$ & $51(17-126)$ & .01 & b \\
\hline
\end{tabular}

Note: Variables are listed as median (interquartile range).

Abbreviations: ANOVA, analysis of variance; TAMI, tibiopedal arterial minimally invasive.

${ }^{a}$ Omnibus $p$-value derived from Kruskal-Wallis ANOVA. Bonferroni post hoc tests were performed among all pairwise comparisons for variables with omnibus $p$-value $<.05$.

bStatistically significant difference between Femoral and Dual Access groups.

${ }^{\text {'S }}$ tatistically significant difference between TAMI and Femoral groups.

${ }^{\mathrm{d} S}$ tatistically significant difference between TAMI and Dual Access groups.

101 were carried using the TAMI technique (retrograde tibial-pedal access) exclusively. Table 1 shows the demographic data. Only the index procedure data was analyzed, and no statistical difference was found in the distribution of mean ages, patients gender, BMI, and comorbidities between groups.

The dual access group included patients with a statistically significant $(p<.001)$ higher median Rutherford class (5 vs. 4) and a lower median number of tibial runoff vessels (1 vs. 2), denoting the group with the most severe and complex disease.
Median fluoroscopy time (14 min), procedure time (64 min), hospital stay ( 0 days; $7.9 \%$ staying $\geq 2$ days), and contrast volume $(50 \mathrm{~mL}$ ) were statistically significantly the lowest in the TAMI access group $(p<.001)$ (Table 2).

Sheath size for vascular access was found to be significantly different across all three cohorts with a $4 \mathrm{Fr}$ sheath most commonly being used for TAMI and dual access and a 5Fr sheath most commonly being used for femoral access. Ultrasound guidance use was significantly higher in the TAMI (99.1\%) and dual access (97.2\%) 
TAB LE 4 Lesion data by access type

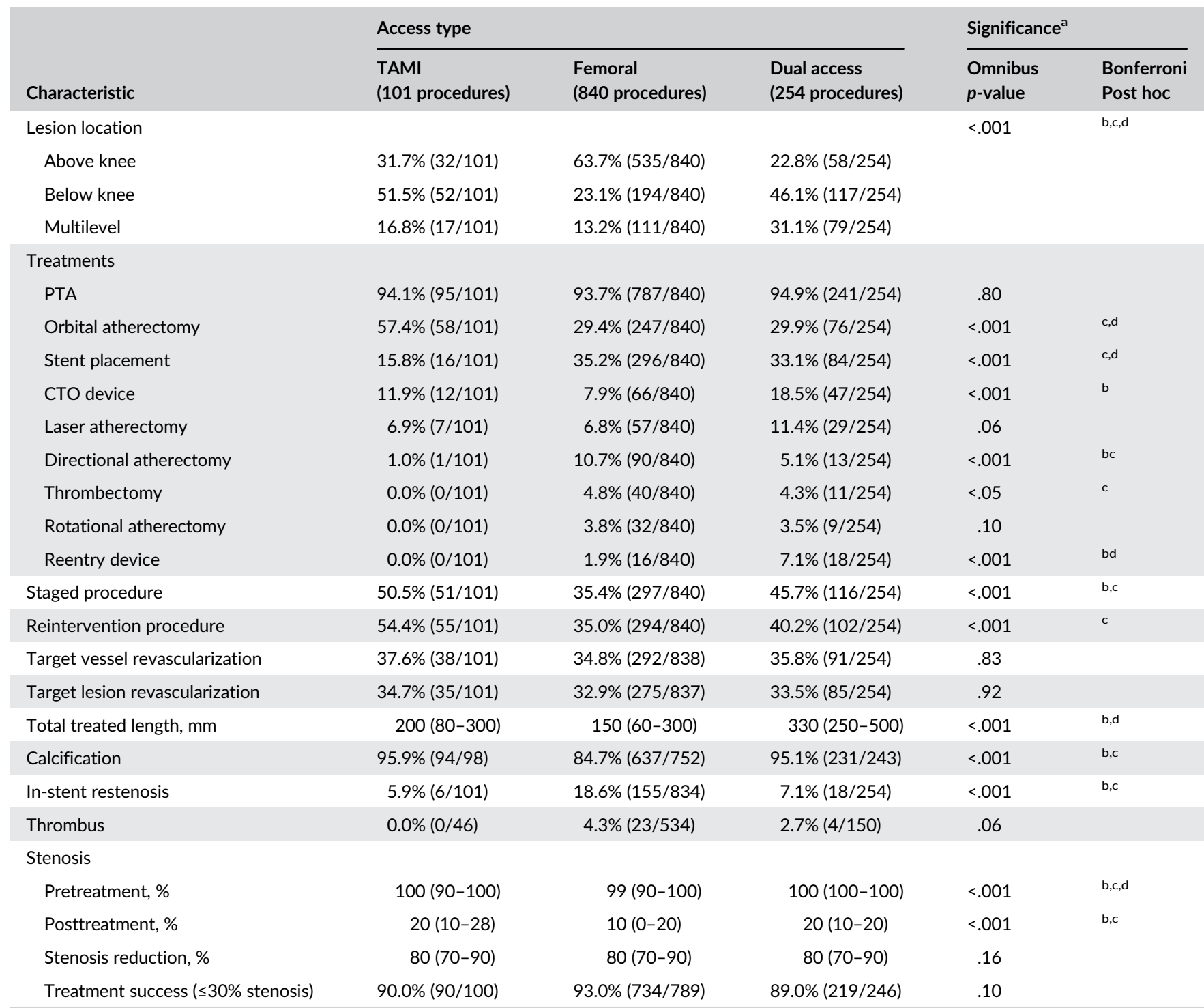

Note: Categorical variables are listed as percent $(n / N)$. Continuous variables are listed as median (interquartile range): $A(B-C)$.

Abbreviations: ANOVA, analysis of variance; TAMI, tibiopedal arterial minimally invasive.

${ }^{a}$ Omnibus $p$-value derived from Fisher's exact test for categorical variables and Kruskal-Wallis ANOVA for continuous variables. Bonferroni post hoc tests were performed among all pairwise comparisons for variables with omnibus $p$-value $<.05$.

${ }^{b}$ Statistically significant difference between Femoral and Dual Access groups.

${ }^{\mathrm{c}}$ Statistically significant difference between TAMI and Femoral groups.

${ }^{\mathrm{d} S}$ Statistically significant difference between TAMI and Dual Access groups.

groups when compared to the femoral access group $(87.7 \%, p<.001)$ (Table 3).

Table 4 shows that there was a significant difference between the three groups with regards to the location of the target lesions treated during the procedures $(p<.001)$. Above the knee lesions were most commonly treated with femoral access, below the knee (BTK) lesions were commonly treated with TAMI access, and multilevel target lesions were routinely treated using dual access (femoral and tibial). Accordingly, significant differences were observed between the TAMI versus the femoral and dual access groups regarding the use of treatment modalities such as orbital atherectomy and stent placement. Orbital atherectomy was used more often with TAMI access (reflecting the predominant treatment of BTK lesions; $p<.001$ ), and stent placement was more commonly used in the common femoral access group (reflecting predominant treatment of supragenicular lesions; $p<.001)$. The use of directional atherectomy was significantly different between femoral (10.7\%), dual access (5.1\%), and TAMI $(1.0 \%)$ groups $(p<.001)$. The use of chronic total occlusion (CTO) and reentry devices showed a statistically significant difference between the femoral and dual access groups $(p<.001)$.

$54.5 \%$ of the TAMI access procedures, were reinterventions. This was statistically significant when compared to the femoral group (35\%; 
TAB LE 5 Complications by access type

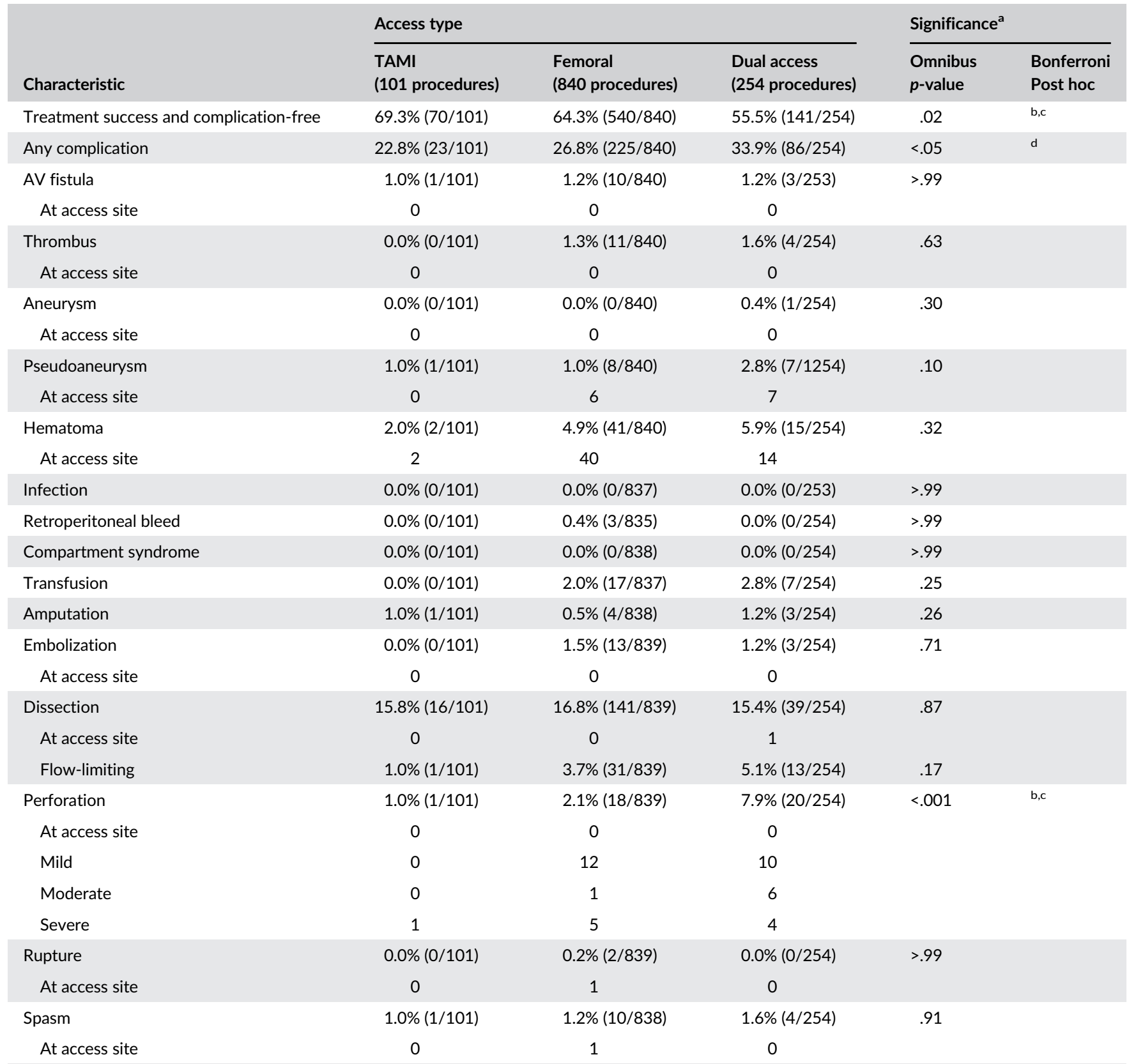

Note: Variables are listed as percent $(n / N)$ or counts.

Abbreviation: TAMI, tibiopedal arterial minimally invasive.

${ }^{a}$ Omnibus $p$-value derived from Fisher's exact test. Bonferroni post hoc tests were performed among all pairwise comparisons for variables with omnibus $p$-value $<.05$.

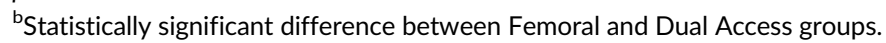

${ }^{\mathrm{c}}$ Statistically significant difference between TAMI and Dual Access groups.

${ }^{d}$ No statistically significant difference among any pairwise comparison.

$p<.001)$. The median total treated lesion length was highest for the dual access cohort $(330 \mathrm{~mm})$ followed by the TAMI cohort $(200 \mathrm{~mm})$. Calcification was similar between the TAMI and dual access groups (95.9\% and $95.1 \%$ respectively), however both were statistically more prevalent than the $84.7 \%$ reported for the femoral group $(p<.001)$.

Pretreatment stenosis was statistically different between all three groups, with the dual access group encompassing CTOs exclusively $(p<.001)$. Posttreatment stenosis was also significantly different between the groups, with the best results achieved in the femoral when compared to both the dual and the TAMI groups $(p<.001$ for each comparison). Treatment success was similar across all groups (Table 4). The analysis of intraprocedural complications revealed that perforations were more significant in the dual access group compared to both the TAMI and common femoral groups $(p<.001)$. The TAMI 
and femoral access groups appeared to have overall, greater treatment success with fewer complications compared to the dual access group $(p=.02)$ (Table 5$)$.

\section{4 | DISCUSSION}

The TAMI technique was originally reported as a retrospective, small, single-center series of patients in a prior publication, which established the safety of the technique. ${ }^{5}$ The PRIME registry is the first $\mathrm{CLI}$ registry in the United States with the sole focus on patients with advanced $P A D$ and $C L I$ diagnoses. The registry examines all aspects of CLI therapy from patient's clinical presentation to revascularization modalities and ultimately clinical outcomes. ${ }^{7}$

CLI patients carry extremely poor outcomes and prognosis. ${ }^{8}$ Unfortunately, major amputation is still perceived as an acceptable form of first line therapy, as shown in a recent study where almost $50 \%$ of patients did not undergo a vascular evaluation prior to amputation. $^{9}$

Patients undergoing a major amputation have a 10-12\% 30-day morbidity / mortality. ${ }^{10} \mathrm{~A}$ significant number never become ambulatory, risk contralateral amputation and suffer a higher mortality rate. ${ }^{11,12}$ A recent NIS (Nationwide Inpatient Sample) study, showed an increased rate of endovascular revascularization coupled with a decrease in surgical revascularization, resulting in a decrease of inhospital mortality and major amputation rates in the United States, ${ }^{5}$ supporting that vascular amputations should not occur without attempting revascularization.

A recent study of 72 thousand Medicare CLI patients, analyzed outcomes of revascularization (surgical and endovascular) vs major amputation, showing that revascularization was more cost effective and produced better outcomes. Survival rates were significantly better in the revascularization arm (38\% endovascular and $40 \%$ surgical) vs. the amputation arm $(23 \%, p<.001) .{ }^{13}$ Patients with advanced PAD and CLI have complex vascular anatomies and comorbidities which can limit successful revascularizations. In an attempt to overcome these hurdles, the TAMI strategy was proposed, ${ }^{6}$ however not validated until now.

In this study, some of our results could be explained by the fact that patients in the femoral access group had the least complex lesions (shortest and least calcified). At the same time, our findings are in agreement with a recent study that looked at СТО lesions and their cap morphology, and showed that severe calcification, cap morphology and lesion length were among the strongest predictors that determined that a retrograde access (used alone [29.6\%] or in combination as dual access [50.6\%]), would be of benefit in $80.2 \%$ of the lesions and more likely to be successful than a conventional, single access with antegrade approach to treat the lesion. ${ }^{14}$ In our study, the dual access group encompassed the most challenging lesions, as all were long (average treated lesion length was $330 \mathrm{~mm}$ ) and severely calcified CTOs. Given the retrospective nature of this analysis, we are unable to comment about how the access strategy was selected. However, combining this data with that generated from the CTOP classification, ${ }^{14}$ it appears safe to state that regardless of the location (supra or infragenicular) of the lesion, long and calcified CTOs (>10 cm in length), would benefit from a dual access strategy. If both the proximal and the distal caps have an antegrade concave morphology and the lesion is $<10 \mathrm{~cm}$ in length, the traditional single access with antegrade approach should suffice. If both caps have a retrograde concavity, and the lesion is $<10 \mathrm{~cm}$ in length, then a straight TAMI approach should be considered.

With regards to the treatment strategy, it appeared to preferentially include orbital and laser atherectomy in the TAMI group. The bail out stenting rate within the TAMI group remained low (15.8\%). The authors believe that the use of atherectomy in these complex and calcific peripheral lesions decreased the rate of bail out stenting, which remains in line with studies examining the use of atherectomy and the limitations of plain balloon angioplasty (especially in infrapopliteal arteries). ${ }^{15,16}$

The overall rate of complications remained low, rendering these procedures and approaches safe. The highest complication rate (despite the overall low number) was seen in the dual access group (as a consequence of femoral access issues). The TAMI group had the lowest complication rates. It also recorded the shortest procedure times, with the lowest radiation exposure (fluoroscopy and cine time), the shortest length of stay, and the lowest amount of contrast used. This is to the best of our knowledge, the first time that all these strategies are compared in the setting of a multicenter study of patients with complex PAD and CLI.

\section{5 | STUDY LIMITATIONS}

Our study represents a retrospective analysis of prospectively collected data and therefore carries the inherent limitation of such design. There were no predetermined selection criteria to include the patients in either group, which along with the high skill set of the operators performing these procedures, could be viewed as potential selection bias. The operators also had intrinsic knowledge of the data from the CTOP classification, ${ }^{14}$ which may have influenced the access selection strategy.

\section{6 | CONCLUSION}

This dataset supports the selection of patients with the aforementioned clinical syndromes and untreated infrapopliteal disease to be approached via a retrograde tibiopedal access as part of a dual access strategy or exclusively via TAMI technique.

TAMI has been shown to be safe (for both patients and operators: lowest complication rate, lowest contrast volume used, lowest radiation exposure), and efficacious (best treatment success rate, shortest length of stay), in the setting of a large multicenter study. Larger prospective and randomized studies may be useful to further validate this approach. 


\section{ACKNOWLEDGMENTS}

The authors thank Larry Miller of Miller Scientific Consulting, Inc, for his statistical support, review, and comments, which have greatly improved the manuscript. The author(s) report receiving the following financial support for the research, authorship, and/or publication of this article: The following companies provided unrestricted research grants to Metro Health-University of Michigan Health to support the PRIME registry: Bard Peripheral Vascular, Terumo Interventional Systems, Boston Scientific, and Cardiovascular Systems, Inc.

\section{CONFLICT OF INTEREST}

Dr Mustapha-Compensated advisor: Bard Peripheral Vascular, Terumo Medical, Boston Scientific and Cardiovascular Systems, Inc. Dr Saab-Compensated advisor: Bard Peripheral Vascular, Terumo Medical, Boston Scientific and Cardiovascular Systems, Inc. Dr JaffNoncompensated advisor: Abbott Vascular, Boston Scientific; Compensated advisor: Medtronic, Philips, Biotronik, Vactronix, Sanofi, BTG Vascular; Equity Investor: Embolitech, Gemini, PQ Bypass. Theresa McGoff, Dr Adams, Dr Mullins, Dr Al-Dadah, Dr Goodney, Dr Khawaja, and Dr Diaz-Sandoval reports no conflicts of interest related to this article.

\section{ORCID}

Larry J. Diaz-Sandoval (D) https://orcid.org/0000-0002-2664-5865

\section{REFERENCES}

1. Fowkes FG, Rudan D, Rudan I, et al. Comparison of global estimates of prevalence and risk factors for peripheral artery disease in 2000 and 2010: a systematic review and analysis. Lancet. 2013;382:13291340.

2. Becker F, Robert-Ebadi H, Ricco JB, et al. Chapter I: definitions, epidemiology, clinical presentation and prognosis. Eur J Vasc Endovasc Surg. 2011;42(suppl 2):S4-S12.

3. Norgren L, Hiatt WR, Dormandy JA, et al. Inter-society consensus for the management of peripheral arterial disease (TASC II). J Vasc Surg. 2007;45(suppl S):S5-S67.

4. Farber A. Chronic limb threatening ischemia. N Engl J Med. 2018 379:171-180.

5. Agarwal S, Sud K, Shishehbor MH. Nationwide trends of hospital admission and outcomes among critical limb ischemia patients: from 2003-2011. J Am Coll Cardiol. 2016;67:1901-1913.

6. Mustapha JA, Saab F, McGoff T, et al. Tibio-pedal arterial minimally invasive retrograde revascularization in patients with advanced peripheral vascular disease: the TAMI technique, original case series. Catheteriz Cardiovasc Interv. 2014;83:987-994.
7. Mustapha JA, Saab F, Diaz-Sandoval L, et al. The peripheral registry of endovascular clinical outcomes (the PRIME registry): interim analysis of the first 328 subjects with critical limb ischemia. Vasc Dis Manag. 2017;14:E55-E66.

8. lida O, Nakamura M, Yamauchi Y, et al. Endovascular treatment for infrainguinal vessels in patients with critical limb ischemia: olive registry, a prospective, multicenter study in Japan with 12-month followup. Circ Cardiovasc Interv. 2013;6:68-76.

9. Swaminathan A, Vemulapalli S, Patel MR, Schuyler Jones W. Lower extremity amputation in peripheral artery disease: improving patient outcomes. Vasc Health Risk Manag. 2014;10:417-424.

10. van Netten JJ, Fortington LV, Hinchliffe RJ, Hijmans JM. Early postoperative mortality after major lower limb amputation: a systematic review of population and regional based studies. Eur J Vasc Endovasc Surg. 2016;51:248-257.

11. Armstrong EJ, Ryan MP, Baker ER, Martinsen BJ, Kotlarz H, Gunnarsson C. Risk of major amputation or death among patients with critical limb ischemia initially treated with endovascular intervention, surgical bypass, minor amputation, or conservative management. J Med Econ. 2017;20:1148-1154.

12. Peacock JM, Keo HH, Duval S, et al. The incidence and health economic burden of ischemic amputation in Minnesota, 2005-2008. Prev Chronic Dis. 2011;8:A141.

13. Mustapha JA, Katzen B, Neville R, et al. Determinants of long-term outcomes and costs in the management of critical limb ischemia: a population-based cohort study. J Am Heart Assoc. 2018;7: e009724.

14. Saab F, Jaff MR, Diaz-Sandoval LJ, et al. Chronic total occlusion crossing approach based on plaque cap morphology: the CTOP classification. J Endovasc Ther. 2018;25:284-291.

15. Mustapha JA, Finton S, Diaz-Sandoval LJ, Saab FA, Miller LE. Percutaneous transluminal angioplasty in patients with infrapopliteal arterial disease. A systematic review and meta-analysis. Circ Cardiovasc Interv. 2016;9:e003468.

16. Shammas NW, Lam R, Mustapha J, et al. Comparison of orbital atherectomy plus balloon angioplasty vs. balloon angioplasty alone in patients with critical limb ischemia: results of the CALCIUM 360 randomized pilot trial. J Endovasc Ther. 2012;19:480-488.

How to cite this article: Mustapha JA, Saab F, McGoff TN, et al. Tibiopedal arterial minimally invasive retrograde revascularization (TAMI) in patients with peripheral arterial disease and critical limb ischemia. On behalf of the Peripheral Registry of Endovascular Clinical Outcomes (PRIME). Catheter Cardiovasc Interv. 2020;95:447-454. https://doi.org/10.1002/ ccd.28639 